\title{
$P$-adic Theta Functions and Solutions of the KP Hierarchy
}

\section{Takashi Ichikawa}

Department of Mathematics, Faculty of Science and Engineering, Saga University, Saga 840, Japan. E-mail address: ichikawa@math.ms.saga-u.ac.jp

Received: 2 August 1994/Accepted: 7 July 1995

\begin{abstract}
Based on Schottky uniformization theory of Riemann surfaces, we construct a universal power series for (Riemann) theta function solutions of the KP hierarchy. Specializing this power series to the coordinates associated with Schottky groups over $p$-adic fields, we show that the $p$-adic theta functions of Mumford curves give solutions of the KP hierarchy.
\end{abstract}

\section{Introduction}

The KP (Kadomtsev-Petvyashvili) hierarchy is a system of infinitely many Lax type partial differential equations,

$$
\frac{\partial L}{\partial t_{n}}=\left[\left(L^{n}\right)_{+}, L\right] ; \quad L=\partial_{x}+\sum_{i=1}^{\infty} u_{i}\left(x, t_{1}, t_{2}, \ldots\right) \partial_{x}^{-i}
$$

$\left(\partial_{x}=\partial / \partial x,\left(L^{n}\right)_{+}\right.$: the nonnegative part of $L^{n}$ for $\left.\partial_{x}\right)$ which includes the KP equation

$$
\frac{3}{4} \frac{\partial^{2} u_{1}}{\partial t_{2}^{2}}-\frac{\partial}{\partial x}\left(\frac{\partial u_{1}}{\partial t_{3}}-\frac{1}{4} \frac{\partial^{3} u_{1}}{\partial x^{3}}-3 u_{1} \frac{\partial u_{1}}{\partial x}\right)=0
$$

Our final goal in this paper is to show that the $p$-adic theta functions of $p$-adic (Mumford) curves give solutions of the KP hierarchy. These solutions are included in algebro-geometric solutions for $p$-adic curves constructed by Krichever $[\mathrm{Kr}]$. However, one cannot express these Krichever solutions in terms of $p$-adic theta functions as is done in $[\mathrm{Kr}]$ for the complex case because there is no theory on curvilinear integrals in $p$-adic analysis.

Our construction of $p$-adic solutions of the KP hierarchy consists of 2 steps: the first step is to obtain a "universal" solution expressed by a formal theta function, and the second step is to specialize this universal solution to $p$-adic solutions. For example, in the genus 1 case, the Weierstrass $\wp$-function

$$
\wp(z)=\frac{1}{z^{2}}+\sum_{u \in L-\{0\}}\left(\frac{1}{(z-u)^{2}}-\frac{1}{u^{2}}\right) \quad(L:=\mathbf{Z}(\pi \tau)+\mathbf{Z} \pi)
$$


for complex numbers $\tau$ with positive imaginary part can be regarded as an element of $\mathbf{Q}[[z, q]][1 / z](q:=\exp (2 \pi \sqrt{-1} \tau))$ which gives a universal elliptic solution of the $\mathrm{KdV}$ (Korteweg-de Vries) hierarchy

$$
\frac{\partial L^{2}}{\partial t_{2 n-1}}=\left[\left(L^{2 n-1}\right)_{+}, L^{2}\right] ; \quad L^{2}=\partial_{x}^{2}+2 u_{1}\left(x, t_{1}, t_{3}, \ldots\right)
$$

and hence for $p$-adic numbers $a$ with $|a|_{p}<1,\left.\wp(z)\right|_{q=a}$ give $p$-adic solutions of $\mathrm{KdV}$. In the genus $\geqq 2$ case, we need Schottky uniformization theory of algebraic curves over local fields for the construction of a universal solution and $p$-adic solutions. It is known in $[\mathrm{H}, \mathrm{Sc}]$ that there exist universal expressions of meromorphic 1-forms and period integrals of Schottky uniformized Riemann surfaces with sufficiently small handles. Based on results in [I], we give power series expansions of these 1-forms and periods with respect to the Koebe coordinates (the fixed points and the eigenvalues of generators of the associated Schottky groups) which, combined with the description given in [Kr] of quasi-periodic solutions of KP in terms of Riemann theta functions, induce a universal power series for solutions of $\mathrm{KP}$ from Riemann surfaces with square roots of canonical bundles. In this expression, the KP hierarchy is reduced to identities between certain formal power series including the universal periods given in [I]. Since pinching handles of Riemann surfaces induces degenerations of the solutions, one can obtain the solitonic degeneration from the universal solution which has been described in [Mum2] for $\mathrm{KdV}$ and in [Go] for KP.

As an application of the universal solution, we will construct formal solutions of the KP hierarchy with coefficients in $p$-adic fields. We show that over $p$-adic fields, the universal 1-forms and periods converge for the Koebe coordinates of any Schottky group, which implies the convergence of the universal solution. Hence this specialization of the universal solution gives solutions of $\mathrm{KP}$ which are seen to be expressed explicitly by the $p$-adic theta functions of Schottky uniformized algebraic curves over $p$-adic fields. It is shown in [Mum1] that an algebraic curve over $p$ adic fields can be Schottky uniformized if and only if this special fiber consists of projective lines, in which case, such a curve is called a Mumford curve. Therefore, we can see that the $p$-adic theta functions of Mumford curves give solutions of the KP hierarchy.

Based on the above result, we propose two problems. The first is to characterize these $p$-adic solutions of the KP hierarchy and the KP equation, which is concerned with a $p$-adic version of the Novikov conjecture mainly studied in [A-D, Mul and Sh]. The second is to construct a theory on Mumford curves "of infinite genus" uniformized by infinitely generated Schottky groups over $p$-adic fields, which will give solutions of the KP hierarchy conjectured to have infinite dimensional orbits.

\section{Riemann Surfaces and the KP Hierarchy}

Let $g$ be a positive integer. We fix a Riemann surface $C$ of genus $g$, a canonical basis $\left\{a_{i}, b_{i}\right\}_{1 \leqq i \leqq g}$ of $H_{1}(C, \mathbf{Z})$ (i.e., $\left.\left(a_{i}, b_{j}\right)=\delta_{i j},\left(a_{i}, a_{j}\right)=\left(b_{i}, b_{j}\right)=0\right)$, a point $P \in C$, and a local coordinate $u$ at $P$ such that $u(P)=0$. We denote the whole data by $X=\left(C,\left\{a_{i}, b_{i}\right\}_{1 \leqq i \leqq g}, P, u\right)$. Then there exists a unique basis $\left\{\omega_{1}, \ldots, \omega_{g}\right\}$ 
of $H^{0}\left(C, \Omega_{C}^{1}\right)$ satisfying $\int_{a_{i}} \omega_{j}=2 \pi \sqrt{-1} \delta_{i j}(i, j=1, \ldots, g)$, and

$$
Z=\left(Z_{i j}\right)_{1 \leqq i, j \leqq g}=\left(\frac{1}{2 \pi \sqrt{-1}} \int_{b_{i}} \omega_{j}\right)_{1 \leqq i, j \leqq g}
$$

is the period matrix of $\left(C,\left\{a_{i}, b_{i}\right\}_{1 \leqq i \leqq g}\right)$. Take $r_{j m} \in \mathbf{C}(j=1, \ldots, g, m \in \mathbf{N})$ such that

$$
\omega_{j}=\sum_{m=1}^{\infty} r_{j m} u^{m-1} d u \quad \text { at } P
$$

For each $n \in \mathbf{N}$, there exists a unique meromorphic 1-form $\omega^{(n)}$ on $C$ satisfying

$$
\begin{gathered}
\omega^{(n)} \text { is holomorphic outside } P \\
\omega^{(n)}=\left(\frac{1}{u^{n+1}}+\sum_{m=1}^{\infty} \frac{q_{n m}}{n} u^{m-1}\right) d u \text { at } P \text { for some } q_{n m} \in \mathbf{C} \\
\int_{a_{i}} \omega^{(n)}=0 \text { for any } i=1, \ldots, g .
\end{gathered}
$$

For $\mathbf{c}=\left(c_{i}\right)_{1 \leqq i \leqq g} \in\left(\mathbf{C}^{\times}\right)^{g}$ and a vector $\vec{z}=\left(z_{i}\right)_{1 \leqq i \leqq g}$ of $g$ indeterminates, we denote the Riemann theta function of $\left(C,\left\{a_{i}, b_{i}\right\}_{1 \leqq i \leqq g}\right)$ by

$$
\Theta(\mathbf{c} \cdot \exp (\vec{z}))=\sum_{\vec{v} \in \mathbf{Z}^{g}}\left\{\prod_{i, j=1}^{g} \exp \left(\pi \sqrt{-1} Z_{i j}\right)^{v_{l} v_{j}} \prod_{i=1}^{g} c_{i}^{v_{l}} \sum_{n=0}^{\infty} \frac{1}{n !}\left(\sum_{i=1}^{g} v_{i} z_{i}\right)^{n}\right\}
$$

where $\vec{v}=\left(v_{i}\right)_{1 \leqq i \leqq g}$. Let $t_{m}(m \in \mathbf{N})$ be indeterminates, and put $\mathbf{t}=\left(t_{1}, t_{2}, t_{3}, \ldots\right)$. Then we define the $\tau$-function $\tau\left(\mathbf{t}, X_{\mathbf{c}}\right)$ as an element of $\mathbf{C}[[\mathbf{t}]]=\mathbf{C}\left[\left[t_{1}, t_{2}, \ldots\right]\right]$ by

$$
\tau\left(\mathbf{t}, X_{\mathbf{c}}\right)=\exp \left(\frac{1}{2} \sum_{n, m=1}^{\infty} q_{n m} t_{n} t_{m}\right) \cdot \Theta\left(\mathbf{c} \cdot \exp \left(\sum_{m=1}^{\infty} t_{m} \vec{r}_{m}\right)\right)
$$

where $\vec{r}_{m}=\left(r_{j m}\right)_{1 \leqq j \leqq g}$. For $\mathbf{c} \in\left(\mathbf{C}^{\times}\right)^{g}$ with $\Theta(\mathbf{c}) \neq 0$, we put

$$
\frac{\tau\left(\mathbf{t}-[z], X_{\mathbf{c}}\right)}{\tau\left(\mathbf{t}, X_{\mathbf{c}}\right)}=1+\sum_{k=1}^{\infty} w_{k}\left(\mathbf{t}, X_{\mathbf{c}}\right) z^{k}
$$

where $[z]=\left(z, z^{2} / 2, z^{3} / 3, \ldots\right)$, and define two micro-differential operators

$$
W\left(\mathbf{t}, X_{\mathbf{c}}\right)=1+\sum_{k=1}^{\infty} w_{k}\left(\mathbf{t}, X_{\mathbf{c}}\right) \partial_{x}^{-k}
$$

and

$$
L\left(\mathbf{t}, X_{\mathbf{c}}\right)=W\left(\mathbf{t}+x, X_{\mathbf{c}}\right) \cdot \partial_{x} \cdot W\left(\mathbf{t}+x, X_{\mathbf{c}}\right)^{-1}
$$

with coefficients in $\mathbf{C}[[x, \mathbf{t}]]$, where $\mathbf{t}+x=\left(t_{1}+x, t_{2}, t_{3}, \ldots\right)$. Then it is known (cf. $[\mathrm{Kr}, \mathrm{S}-\mathrm{S}])$ that $L\left(\mathbf{t}, X_{\mathbf{c}}\right)$ satisfies the KP hierarchy

$$
\frac{\partial L}{\partial t_{n}}=\left[\left(L^{n}\right)_{+}, L\right] \quad(n \in \mathbf{N}) .
$$


In particular,

$$
u_{1}\left(x, t_{2}, t_{3}\right)=\frac{\partial^{2}}{\partial x^{2}} \log \Theta\left(\mathbf{c} \cdot \exp \left(x \vec{r}_{1}+t_{2} \vec{r}_{2}+t_{3} \vec{r}_{3}\right)\right)+q_{11}
$$

satisfies the KP equation

$$
\frac{3}{4} \frac{\partial^{2} u_{1}}{\partial t_{2}^{2}}-\frac{\partial}{\partial x}\left(\frac{\partial u_{1}}{\partial t_{3}}-\frac{1}{4} \frac{\partial^{3} u_{1}}{\partial x^{3}}-3 u_{1} \frac{\partial u_{1}}{\partial x}\right)=0
$$

\section{Schottky Uniformization}

2.1. Let $K$ be $\mathbf{C}$ or a complete nonarchimedean valuation field with multiplicative valuation || . Let $P G L_{2}(K)$ act on $\mathbf{P}^{1}(K)$ by the Möbius transformation. A subgroup $\Gamma$ of $P G L_{2}(K)$ is called a Schottky group of rank $g$ over $K$ if there exist its free generators $\gamma_{1}, \ldots, \gamma_{g}$ and $2 g$ open domains bounded by Jordan curves if $K=\mathbf{C}$ (resp. $2 g$ open disks if $K$ is a nonarchimedean valuation field) $D_{ \pm 1}, \ldots, D_{ \pm g} \subset \mathbf{P}^{1}(K)$ such that

$$
\bar{D}_{i} \cap \bar{D}_{j}=\emptyset(i \neq j), \quad \gamma_{k}\left(\mathbf{P}^{1}(K)-D_{-k}\right)=\bar{D}_{k}(k=1, \ldots, g),
$$

and then $\Gamma$ is called to be marked if selecting such a sequence $\gamma_{1}, \ldots, \gamma_{g}$. Then for each $k=1, \ldots, g$, we can take uniquely $\alpha_{ \pm k} \in D_{ \pm k}$ and $\beta_{k} \in K^{\times}$such that

$$
\gamma_{k}=\left(\begin{array}{cc}
\alpha_{k} & \alpha_{-k} \\
1 & 1
\end{array}\right)\left(\begin{array}{cc}
1 & 0 \\
0 & \beta_{k}
\end{array}\right)\left(\begin{array}{cc}
\alpha_{k} & \alpha_{-k} \\
1 & 1
\end{array}\right)^{-1} \bmod \left(K^{\times}\right),
$$

i.e.,

$$
\frac{\gamma_{k}(z)-\alpha_{k}}{\gamma_{k}(z)-\alpha_{-k}}=\beta_{k} \frac{z-\alpha_{k}}{z-\alpha_{-k}} \quad\left(z \in \mathbf{P}^{1}(K)\right) \text {. }
$$

We call $\left(\alpha_{ \pm k}, \beta_{k}\right)_{1 \leqq k \leqq g}$ the Koebe coordinates of $\left(\Gamma ; \gamma_{1}, \ldots, \gamma_{g}\right)$. A Schottky group $\Gamma$ is called classical if we can take $D_{ \pm k}(k=1, \ldots, g)$ as open disks (hence all Schottky groups over nonarchimedean valuation fields are automatically classical (cf. $[\mathrm{G}-\mathrm{v} . \mathrm{d} . \mathrm{P}]))$. Then there exist $\mu_{ \pm 1}, \ldots, \mu_{ \pm g}<1$ such that

$$
D_{ \pm k}=\left\{z \in \mathbf{P}^{1}(K) ;\left|z-\alpha_{ \pm k}\right|<\mu_{ \pm k}\left|z-\alpha_{\mp k}\right|\right\} \quad(k=1, \ldots, g) .
$$

In particular,

$$
\left|\beta_{k}\right|<\min \left\{\left|\left[\alpha_{k}, \alpha_{-k} ; \alpha_{i}, \alpha_{j}\right]\right| ; i, j \neq \pm k\right\} \quad(k=1, \ldots, g),
$$

where

$$
[a, b ; c, d]=\frac{(a-c)(b-d)}{(a-d)(b-c)}
$$

denotes the cross ratio of four points. Put

$$
F_{\Gamma}=\mathbf{P}^{1}(K)-\bigcup_{k=1}^{g}\left(D_{k} \cup \overline{D_{-k}}\right), \quad H_{\Gamma}=\bigcup_{\gamma \in \Gamma} \gamma\left(F_{\Gamma}\right) .
$$

Then $\Gamma$ acts on $H_{\Gamma}$ freely and properly discontinuously, and the quotient $K$-analytic space $C_{\Gamma}=H_{\Gamma} / \Gamma$ is obtained from $\mathbf{P}^{1}(K)-\cup_{k=1}^{g} D_{ \pm k}$ by identifying $\partial D_{k}$ and $\partial D_{-k}$ via $\gamma_{k}(k=1, \ldots, g)$. It is known that the $K$-analytic space $C_{\Gamma}$ has naturally 
the structure of a proper and smooth algebraic curve of genus $g$ over $K$. Moreover, it is known that any Riemann surface can be Schottky uniformized, and that an algebraic curve over a nonarchimedean valuation field can be Schottky uniformized if and only if this curve is a Mumford curve, i.e., the special fiber consists of projective lines (cf. [Ko, Mum1]). Let $S_{K}$ be the subset of $\left(\mathbf{P}^{1}(K) \times\right.$ $\left.\mathbf{P}^{1}(K) \times K^{\times}\right)^{g}$ consisting of the Koebe coordinates of all marked Schottky groups over $K$. For $k=-1, \ldots,-g$, put $\beta_{k}=\beta_{-k}$ and $\gamma_{k}=\left(\gamma_{-k}\right)^{-1}$. Then $\gamma_{k}$ satisfies (2.1) also.

2.2. Proposition. Let $\left(\Gamma ; \gamma_{1}, \ldots, \gamma_{g}\right)$ be a marked Schottky group over $K$ with Koebe coordinates $\left(\alpha_{ \pm k}, \beta_{k}\right)_{1 \leqq k \leqq g}$, and let $\Gamma^{\prime}$ be the Schottky group generated by $\gamma_{1}, \ldots, \gamma_{g-1}$. Then under $\bar{\beta}_{g} \rightarrow 0, C_{\Gamma}$ becomes the degenerate algebraic curve $C^{\prime}$ obtained by identifying $\alpha_{g}$ and $\alpha_{-g}$ in $C_{\Gamma^{\prime}}$.

Proof. For any $\varepsilon \in K^{\times}$with $|\varepsilon| \leqq 1$, let $D_{ \pm g}(\varepsilon)$ be the domains containing $\alpha_{ \pm g}$ given by

$$
D_{ \pm g}(\varepsilon)=\left\{z \in \mathbf{P}^{1}(K) ;\left|z-\alpha_{ \pm g}\right|<\sqrt{|\varepsilon|} \mu_{ \pm g}\left|z-\alpha_{\mp g}\right|\right\},
$$

and put

$$
\gamma_{g}(\varepsilon)=\left(\begin{array}{cc}
\alpha_{g} & \alpha_{-g} \\
1 & 1
\end{array}\right)\left(\begin{array}{cc}
1 & 0 \\
0 & \varepsilon \beta_{g}
\end{array}\right)\left(\begin{array}{cc}
\alpha_{g} & \alpha_{-g} \\
1 & 1
\end{array}\right)^{-1} \bmod \left(K^{\times}\right) .
$$

Then $\gamma_{g}(\varepsilon)\left(\partial D_{-g}(\varepsilon)\right)=\partial D_{g}(\varepsilon)$. Let $\Gamma(\varepsilon)$ be the subgroup of $P G L_{2}(K)$ generated by $\gamma_{1}, \ldots, \gamma_{g-1}$ and $\gamma_{g}(\varepsilon)$. Then $\Gamma(\varepsilon)$ is a Schottky group of rank $g$ and $C_{\Gamma(\varepsilon)}$ is isomorphic to the $K$-analytic space obtained by identifying $\partial D_{k}$ and $\partial D_{-k}$ via $\gamma_{k}(k=1, \ldots, g-1), \partial D_{g}(\varepsilon)$ and $\partial D_{-g}(\varepsilon)$ via $\gamma_{g}(\varepsilon)$. Therefore, if $|\varepsilon| \rightarrow 0$, then $D_{ \pm g}(\varepsilon)$ tend to $\alpha_{ \pm g}$, and hence $C_{\Gamma(\varepsilon)}$ becomes $C^{\prime}$.

2.3. Let $K=\mathbf{C}$, and let the notation be as in 2.1. Taking the conjugation $\gamma_{i} \mapsto$ $\rho \gamma_{i} \rho^{-1}(i=1, \ldots, g)$ by a certain $\rho \in P G L_{2}(\mathbf{C})$, we may assume that $\infty \in F_{\Gamma}$ which implies that $\alpha_{ \pm k} \neq \infty(k=1, \ldots, g)$. For each $i=1, \ldots, g$, let $a_{i}$ be the closed path $\partial D_{i}$ counterclockwise oriented, and let $b_{i}$ be an oriented path in $F_{\Gamma}$ from a certain point $x_{i}$ of $\partial D_{-i}$ to $\gamma_{i}\left(x_{i}\right)$ such that $b_{i} \cap b_{j}=\emptyset(i \neq j)$. Then $\left\{a_{i}, b_{i}\right\}_{1 \leqq i \leqq g}$ becomes a canonical basis of $H_{1}\left(C_{\Gamma}, \mathbf{Z}\right)$. Let $p$ be a point of $F_{\Gamma}-\{\infty\}$, let $P$ be the point of $C_{\Gamma}$ corresponding to $p$, and put $u=z-p$. Assume that $\beta_{1}, \ldots, \beta_{g}$ are sufficiently small. Then we express $\omega^{(n)}, \omega_{j}$, and $Z_{i j}$ explicitly for $\left(C_{\Gamma},\left\{a_{i}, b_{i}\right\}_{1 \leqq i \leqq g}, P, u\right)$ according to [H]. It is shown in [Sc] (see also [A]) that $\Sigma_{\gamma \in \Gamma}\left|\gamma^{\prime}(z)\right|$ is uniformly convergent on $H_{\Gamma}-\cup_{\gamma \in \Gamma} \gamma(\infty)$ under the above assumption. Hence for each integer $n \geqq 0$, one can define a meromorphic 1 -form

$$
\omega^{(n)}=\sum_{\gamma \in \Gamma} \frac{\gamma^{\prime}(z)}{(\gamma(z)-p)^{n+1}} d z
$$

on $H_{\Gamma}$. Since $\omega^{(n)}$ is $\Gamma$-invariant, this induces a meromorphic 1-form on $C_{\Gamma}$ which we denote by the same symbol, and a term-by-term integration shows that $\int_{a_{l}} \omega^{(n)}=0(i=1, \ldots, g)$. It is easy to see that $\omega^{(0)}$ is holomorphic except for simple poles of residue 1 (resp. -1 ) at $P$ (resp. $\infty$ ), and that $\omega^{(n)}(n \geqq 1)$ satisfy (1.1) -(1.3). For each $j=1, \ldots, g$, let $\omega_{j}$ be the holomorphic 1 -form on $C_{\Gamma}$ such that $\int_{a_{i}} \omega_{j}=2 \pi \sqrt{-1} \delta_{i j}(i=1, \ldots, g)$. Then by the classical period relation,

$$
\int_{\infty}^{P} \omega_{j}=\int_{b_{j}} \omega^{(0)}=\int_{x_{j}}^{\gamma_{j}\left(x_{j}\right)} \omega^{(0)}
$$


Hence

$$
\begin{aligned}
\omega_{j} & =\sum_{\gamma \in \Gamma}\left(\frac{1}{z-\left(\gamma \gamma_{j}\right)\left(x_{j}\right)}-\frac{1}{z-\gamma\left(x_{j}\right)}\right) d z \\
& =\sum_{\gamma \in \Gamma_{j}}\left(\frac{1}{z-\gamma\left(\alpha_{j}\right)}-\frac{1}{z-\gamma\left(\alpha_{-j}\right)}\right) d z \\
& =\sum_{m=1}^{\infty} \sum_{k=1}^{m} \sum_{\gamma \in \Gamma_{j}}\left(\frac{\gamma\left(\alpha_{j}\right)-\gamma\left(\alpha_{-j}\right)}{\left(\gamma\left(\alpha_{j}\right)-p\right)^{k}\left(\gamma\left(\alpha_{-j}\right)-p\right)^{m+1-k}}\right) u^{m-1} d u,
\end{aligned}
$$

where $\Gamma_{j}(j=1, \ldots, g)$ denotes a complete set of representatives of the cosets $\Gamma /\left\langle\gamma_{j}\right\rangle$. Since

$$
Z_{i j}=\frac{1}{2 \pi \sqrt{-1}} \int_{b_{i}} \omega_{j}=\frac{1}{2 \pi \sqrt{-1}} \int_{x_{i}}^{\gamma_{i}\left(x_{i}\right)} \omega_{j}(i, j=1, \ldots, g),
$$

taking appropriate branches of the logarithm, we have

$$
\begin{aligned}
Z_{i j} & =\frac{1}{2 \pi \sqrt{-1}} \sum_{\gamma \in \Gamma_{j}} \log \left(\left[\gamma_{i}(x), x ; \gamma\left(\alpha_{j}\right), \gamma\left(\alpha_{-j}\right)\right]\right) \\
& =\frac{1}{2 \pi \sqrt{-1}} \sum_{\gamma \in \Gamma_{i j}} \log \left(\psi_{i j}(\gamma)\right)
\end{aligned}
$$

where $\Gamma_{i j}(i, j=1, \ldots, g)$ denotes a complete set of representatives of the cosets $\left\langle\gamma_{i}\right\rangle \backslash \Gamma /\left\langle\gamma_{j}\right\rangle$, and $\psi_{i j}$ is the map $\Gamma_{i j} \rightarrow \mathbf{C}^{\times}$given by

$$
\psi_{i j}(\gamma)= \begin{cases}\beta_{i} & \left(\text { if } i=j \text { and } \gamma \in\left\langle\gamma_{i}\right\rangle\right) \\ {\left[\alpha_{i}, \alpha_{-i} ; \gamma\left(\alpha_{j}\right), \gamma\left(\alpha_{-j}\right)\right]} & \text { (otherwise). }\end{cases}
$$

For each $i, j=1, \ldots, g$, put $p_{i j}=\exp \left(2 \pi \sqrt{-1} Z_{i j}\right)$. Then

$$
p_{i j}=\prod_{\gamma \in \Gamma_{i j}} \psi_{i j}(\gamma)
$$

We note that in [M-D], the above $\omega_{j}$ and $p_{i j}$ are obtained as holomorphic 1 -forms and multiplicative periods of Schottky uniformized Mumford curves (cf. Corollary 4.4).

\section{Universal Solution of KP}

3.1. Let $x_{ \pm k}, y_{k}(k=1, \ldots, g), p$, and $z$ be variables, and put $u=z-p$. Let $A$ be the ring of formal power series over $\mathbf{Z}\left[x_{k}, \Pi_{i<j} 1 /\left(x_{i}-x_{j}\right)\right](i, j, k \in\{ \pm 1, \ldots, \pm g\})$ with variables $y_{1}, \ldots, y_{g}$, i.e.,

$$
A=\mathbf{Z}\left[x_{k}, \prod_{i<j} \frac{1}{x_{i}-x_{j}}\right]\left[\left[y_{1}, \ldots, y_{g}\right]\right]
$$

and put

$$
A_{p}=A\left[\prod_{k=1}^{g} \frac{1}{\left(x_{k}-p\right)\left(x_{-k}-p\right)}\right] \text {. }
$$


Let $I$ be the ideal of $A$ generated by $y_{1}, \ldots, y_{g}$. For each $k= \pm 1, \ldots, \pm g$, put $y_{k}=y_{|k|}$, and let $f_{k}$ be the element of $G L_{2}(\Omega)(\Omega:$ the quotient field of $A)$ given by

$$
f_{k}=\left(\begin{array}{cc}
x_{k} & x_{-k} \\
1 & 1
\end{array}\right)\left(\begin{array}{cc}
1 & 0 \\
0 & y_{k}
\end{array}\right)\left(\begin{array}{cc}
x_{k} & x_{-k} \\
1 & 1
\end{array}\right)^{-1} .
$$

3.2. Proposition. For any $f=f_{k(1)} f_{k(2)} \cdots f_{k(l)}$ satisfying $k(s) \neq-k(s+1)$ $(s=1, \ldots, l-1)$, put $y=y_{k(1)} y_{k(2)} \cdots y_{k(l)}$. Then we have

(a) $f\left(x_{j}\right) \in A$, and $f\left(x_{j}\right) \in x_{k(1)}+I$ for $f \notin\left\langle f_{j}\right\rangle$,

(b) $f\left(x_{j}\right)-f\left(x_{-j}\right) \in y A$ for $j \neq \pm k(l)$,

(c) $\left[x_{i}, x_{-i} ; f\left(x_{j}\right), f\left(x_{-j}\right)\right] \in 1+y A$ for $i \neq \pm k(1), j \neq \pm k(l)$,

(d) $\frac{f^{\prime}(z)}{(f(z)-p)^{n+1}} \in y\left(A_{p}[[u]]\right)$.

Proof. (a) We prove this by induction on $l$. Assume that $f \notin\left\langle f_{j}\right\rangle$ and $f\left(x_{j}\right)=$ $x_{k(1)}+a$ for some $a \in I$. Then for any $i \neq-k(1)$,

$$
\left(f\left(x_{j}\right)-x_{-i}\right)^{-1}=\left\{\left(x_{k(1)}-x_{-i}\right)\left(1+\frac{a}{x_{k(1)}-x_{-i}}\right)\right\}^{-1} \in A .
$$

Hence

$$
\left(f_{i} f\right)\left(x_{j}\right)=\left\{x_{i}-\frac{\left(f\left(x_{j}\right)-x_{i}\right) x_{-i} y_{i}}{f\left(x_{j}\right)-x_{-i}}\right\}\left\{1-\frac{\left(f\left(x_{j}\right)-x_{i}\right) y_{i}}{f\left(x_{j}\right)-x_{-i}}\right\}^{-1}
$$

belongs to $x_{i}+I$. Assume that $f \in\left\langle f_{j}\right\rangle$. Then $f\left(x_{j}\right)=x_{j}$, and hence for any $i \neq \pm j,\left(f_{i} f\right)\left(x_{j}\right) \in x_{i}+I$.

(b) We prove this by induction on $l$. Assume that $j \neq \pm k(l)$ and $f\left(x_{j}\right)-$ $f\left(x_{-j}\right) \in y A$. Then by (a), $f\left(x_{j}\right)=x_{k(1)}+b$ for some $b \in I$, and hence for any $i \neq-k(1)$,

$$
\left\{f\left(x_{j}\right)-x_{-i}-y_{i}\left(f\left(x_{j}\right)-x_{i}\right)\right\}^{-1}=\left\{\left(x_{k(1)}-x_{-i}\right)+\left(b-y_{i}\left(f\left(x_{j}\right)-x_{i}\right)\right)\right\}^{-1}
$$

belongs to $A$. Similarly, $\left\{f\left(x_{-j}\right)-x_{-i}-y_{i}\left(f\left(x_{-j}\right)-x_{i}\right)\right\}^{-1}$ belongs to $A$. Hence

$$
\begin{aligned}
& \left(f_{i} f\right)\left(x_{j}\right)-\left(f_{i} f\right)\left(x_{-j}\right) \\
& \quad=\frac{\left(x_{i}-x_{-i}\right)^{2}\left(f\left(x_{j}\right)-f\left(x_{-j}\right)\right) y_{i}}{\left\{f\left(x_{j}\right)-x_{-i}-y_{i}\left(f\left(x_{j}\right)-x_{i}\right)\right\}\left\{f\left(x_{-j}\right)-x_{-i}-y_{i}\left(f\left(x_{-j}\right)-x_{l}\right)\right\}}
\end{aligned}
$$

belongs to $y y_{i} A$.

(c) By (a), $\left(f\left(x_{j}\right)-x_{-i}\right)^{-1}$ and $\left(f\left(x_{-j}\right)-x_{i}\right)^{-1}$ belong to $A$. Therefore, by (b),

$$
\left[x_{i}, x_{-i} ; f\left(x_{j}\right), f\left(x_{-j}\right)\right]=1+\frac{\left(x_{i}-x_{-i}\right)\left(f\left(x_{j}\right)-f\left(x_{-j}\right)\right)}{\left(f\left(x_{j}\right)-x_{-i}\right)\left(f\left(x_{-j}\right)-x_{i}\right)}
$$

belongs to $1+y A$.

(d) Put

$$
f=\left(\begin{array}{ll}
a_{f} & b_{f} \\
c_{f} & d_{f}
\end{array}\right)
$$


Then

$$
\frac{f^{\prime}(z)}{(f(z)-p)^{n+1}}=\frac{\left(a_{f} d_{f}-b_{f} c_{f}\right)\left\{c_{f} p+d_{f}+c_{f} u\right\}^{n-1}}{\left\{\left(a_{f} p+b_{f}-c_{f} p^{2}-d_{f} p\right)+\left(a_{f}-c_{f} p\right) u\right\}^{n+1}}
$$

Since

$$
f_{k}=\frac{1}{x_{k}-x_{-k}}\left\{\left(\begin{array}{cc}
x_{k} & -x_{k} x_{-k} \\
1 & -x_{-k}
\end{array}\right)-\left(\begin{array}{cc}
x_{-k} & -x_{-k} x_{k} \\
1 & -x_{k}
\end{array}\right) y_{k}\right\}
$$

and

$$
\left(\begin{array}{cc}
\alpha & -\alpha \beta \\
1 & -\beta
\end{array}\right)\left(\begin{array}{cc}
\gamma & -\gamma \delta \\
1 & -\delta
\end{array}\right)=(\gamma-\beta)\left(\begin{array}{cc}
\alpha & -\alpha \delta \\
1 & -\delta
\end{array}\right),
$$

$a_{f}, b_{f}, c_{f}$ and $d_{f}$ belong to $A$, and these constant terms are $x_{k(1)} t,-x_{k(1)} x_{-k(l)} t, t$ and $-x_{-k(l)} t$ respectively, where

$$
t=\frac{\prod_{s=2}^{l}\left(x_{k(s)}-x_{-k(s-1)}\right)}{\prod_{s=1}^{l}\left(x_{k(s)}-x_{-k(s)}\right)} \in A^{\times} .
$$

Hence the constant terms of $c_{f} p+d_{f}$ and $a_{f} p+b_{f}-c_{f} p^{2}-d_{f} p$ are $-\left(x_{-k(l)}-\right.$ $p) t \in A_{p}^{\times}$and $-\left(x_{k(1)}-p\right)\left(x_{-k(l)}-p\right) t \in A_{p}^{\times}$respectively. This implies (d).

3.3. Put $\varphi_{k}=f_{k} \bmod \left(\Omega^{\times}\right) \in P G L_{2}(\Omega)$ for $k= \pm 1, \ldots, \pm g$, and let $\Phi$ be the free subgroup of $P G L_{2}(\Omega)$ with generators $\varphi_{1}, \ldots, \varphi_{g}$. Then $\varphi_{-k}=\varphi_{k}^{-1}$ and $\varphi_{k}(z)=$ $f_{k}(z)$. Let $\Phi_{j}$ and $\Phi_{i j}$ be the subsets of $\Phi$ given by

$$
\begin{aligned}
\Phi_{j} & =\left\{\varphi_{k(1)} \cdots \varphi_{k(l)} ; k(s) \neq-k(s+1), k(l) \neq \pm j\right\}, \\
\Phi_{i j} & =\left\{\varphi_{k(1)} \cdots \varphi_{k(l)} ; k(s) \neq-k(s+1), k(1) \neq \pm i, k(l) \neq \pm j\right\} .
\end{aligned}
$$

Then $\Phi_{j}$ (resp. $\Phi_{i j}$ ) is a complete set of representatives of the cosets $\Phi /\left\langle\varphi_{j}\right\rangle$ (resp. $\left.\left\langle\varphi_{i}\right\rangle \backslash \Phi /\left\langle\varphi_{j}\right\rangle\right)$. Hence by Proposition 3.2, one can define two 1-forms with coefficients in $A_{p}$ and an element of $A$ as follows:

$$
\begin{aligned}
\Omega^{(n)} & =\sum_{\varphi \in \Phi} \frac{\varphi^{\prime}(z)}{(\varphi(z)-p)^{n+1}} d u(n \geqq 0), \\
\Omega_{j} & =\sum_{m=1}^{\infty} \sum_{k=1}^{m} \sum_{\varphi \in \Phi_{J}} \frac{\left(\varphi\left(x_{j}\right)-\varphi\left(x_{-j}\right)\right) u^{m-1}}{\left(\varphi\left(x_{j}\right)-p\right)^{k}\left(\varphi\left(x_{-j}\right)-p\right)^{m+1-k}} d u(j=1, \ldots, g), \\
P_{i j} & =\prod_{\varphi \in \Phi_{i j}} \psi_{i j}(\varphi)(i, j=1, \ldots, g),
\end{aligned}
$$

where

$$
\psi_{i j}(\varphi)= \begin{cases}y_{i} & \left(\text { if } i=j \text { and } \varphi \in\left\langle\varphi_{i}\right\rangle\right) \\ {\left[x_{i}, x_{-i} ; \varphi\left(x_{j}\right), \varphi\left(x_{-j}\right)\right]} & \text { (otherwise) }\end{cases}
$$

We introduce $g$ variables $y_{i}^{1 / 2}(i=1, \ldots, g)$ which are square roots of $y_{i}$, and define square roots $P_{l i}^{1 / 2} \in A\left[y_{1}^{1 / 2}, \ldots, y_{g}^{1 / 2}\right] \hat{\otimes}_{\mathbf{Z}} \mathbf{Q}$ of $P_{i i}(i=1, \ldots, g)$ by

$$
P_{i i}^{1 / 2}=y_{i}^{1 / 2} \sum_{n=0}^{\infty}\left(\begin{array}{c}
1 / 2 \\
n
\end{array}\right)\left\{\prod_{\varphi \in \Phi_{i i}-\{1\}} \psi_{i i}(\varphi)-1\right\}^{n} .
$$


Then for two sequences $\mathbf{w}=\left(w_{i}\right)_{1 \leqq i \leqq g}$ and $\vec{z}=\left(z_{i}\right)_{1 \leqq i \leqq g}$ of $g$ indeterminates, the universal theta function is defined by

$$
\Theta(\mathbf{w} \cdot \exp (\vec{z}))=\sum_{\vec{v} \in \mathbf{Z}^{g}}\left\{\prod_{i=1}^{g} P_{i i}^{v_{i}^{2} / 2} \prod_{i<j} P_{i j}^{v_{i} v_{j}} \prod_{i=1}^{g} w_{i}^{v_{i}} \sum_{n=0}^{\infty} \frac{1}{n !}\left(\sum_{i=1}^{g} v_{i} z_{i}\right)^{n}\right\} .
$$

Let $Q_{n m}$ and $R_{j m}$ be the elements of $A_{p}$ such that

$$
\begin{aligned}
\Omega^{(n)} & =\left(\frac{1}{u^{n+1}}+\sum_{m=1}^{\infty} \frac{Q_{n m}}{n} u^{m-1}\right) d u(n \geqq 0), \\
\Omega_{j} & =\sum_{m=1}^{\infty} R_{j m} u^{m-1} d u(j=1, \ldots, g),
\end{aligned}
$$

and put $\vec{R}_{m}=\left(R_{j m}\right)_{1 \leqq j \leqq g}$. Then we define the universal $\tau$-function by

$$
\tau(\mathbf{t})=\exp \left(\frac{1}{2} \sum_{n, m=1}^{\infty} Q_{n m} t_{n} t_{m}\right) \cdot \Theta\left(\mathbf{w} \cdot \exp \left(\sum_{m=1}^{\infty} t_{m} \vec{R}_{m}\right)\right)
$$

which is an element of

$$
B=A_{p}\left[y_{1}^{1 / 2}, \ldots, y_{g}^{1 / 2}, w_{1}^{ \pm 1}, \ldots, w_{g}^{ \pm 1}\right] \hat{\otimes}_{\mathbf{z}} \mathbf{Q}[[\mathbf{t}]] .
$$

Since $\tau(\mathbf{t})-1$ belongs to the ideal of $B$ generated by $y_{1}^{1 / 2}, \ldots, y_{g}^{1 / 2}$, as in (1.4)(1.6), $\tau(\mathbf{t})$ defines a micro-differential operator $L(\mathbf{t})$ with coefficients in $B[[x]]$.

\subsection{Theorem.}

(a) Let $\left(\Gamma ; \gamma_{1}, \ldots, \gamma_{g}\right)$ be a marked Schottky group over $\mathbf{C}$ with Koebe coordinates $\left(\alpha_{ \pm k}, \beta_{k}\right)_{1 \leqq k \leqq g}$, and let $X=\left(C_{\Gamma},\left\{a_{i}, b_{i}\right\}_{1 \leqq i \leqq g}, P, u\right)$ be as in 2.3 with period matrix $\left(Z_{i j}\right)_{1 \leqq i, j \leqq g}$. Assume that $\beta_{1}, \ldots, \beta_{g}$ are sufficiently small, and take $\beta_{1}^{1 / 2}, \ldots, \beta_{g}^{1 / 2}$ such that

$$
\beta_{i}^{1 / 2} \sum_{n=0}^{\infty}\left(\begin{array}{c}
1 / 2 \\
n
\end{array}\right)\left\{\prod_{\gamma \in \Gamma_{i i}-\{1\}} \psi_{i i}(\gamma)-1\right\}^{n}=\exp \left(\pi \sqrt{-1} Z_{i i}\right) .
$$

Then for any $\mathbf{c}=\left(c_{i}\right)_{1 \leqq i \leqq g} \in\left(\mathbf{C}^{\times}\right)^{g}$ with $\Theta(\mathbf{c}) \neq 0$,

$$
\left.L(\mathbf{t})\right|_{x_{k}=\alpha_{k}, y_{k}^{1 / 2}=\beta_{k}^{1 / 2}, w_{k}=c_{k}}=L\left(\mathbf{t}, X_{\mathbf{c}}\right) .
$$

(b) L(t) satisfies the KP hierarchy (1.7). In particular,

$$
u_{1}\left(x, t_{2}, t_{3}\right)=\frac{\partial^{2}}{\partial x^{2}} \log \Theta\left(\mathbf{w} \cdot \exp \left(x \vec{R}_{1}+t_{2} \vec{R}_{2}+t_{3} \vec{R}_{3}\right)\right)+Q_{11}
$$

satisfies the KP equation (1.8).

Proof. Assertion (a) follows from the definition of $L(\mathbf{t})$. Hence as seen in Sect. 1, $\partial L(\mathbf{t}) / \partial t_{n}$ and $\left[\left(L(\mathbf{t})^{n}\right)_{+}, L(\mathbf{t})\right]$ coincide for $x_{k}=\alpha_{k}, y_{k}^{1 / 2}=\beta_{k}^{1 / 2}, w_{k}=c_{k}, \quad p \in F_{\Gamma}$ if $\left(\alpha_{ \pm k}, \beta_{k}\right)_{1 \leqq k \leqq g} \in S_{K}, \beta_{1}, \ldots, \beta_{g}$ are sufficiently small and $\mathbf{c}$ is generic (i.e., $\Theta(\mathbf{c}) \neq 0$ ). Therefore, $\partial L(\mathbf{t}) / \partial t_{n}=\left[\left(L(\mathbf{t})^{n}\right)_{+}, L(\mathbf{t})\right]$. 
3.5. By Proposition 2.2, for a marked Schottky group $\left(\Gamma ; \gamma_{1}, \ldots, \gamma_{g}\right)$ with Koebe coordinates $\left(\alpha_{ \pm k}, \beta_{k}\right)_{1 \leqq k \leqq g}$, if $\beta_{1}, \ldots, \beta_{g} \rightarrow 0$, then $C_{\Gamma}$ becomes a degenerate curve obtained from $\mathbf{P}^{1}(K)$ by identifying $\alpha_{k}$ and $\alpha_{-k}(k=1, \ldots, g)$. By Proposition 3.2,

$$
\left.\tau(\mathbf{t})\right|_{y_{1}=\cdots=y_{g}=0}=1 \text {, }
$$

and hence $\left.L(\mathbf{t})\right|_{y_{1}=\cdots=y_{g}=0}=\partial_{x}$ which we call the trivial degeneration. On the other hand, generalizing a modified theta function in [Go, Mum2], we define

$$
\Theta_{\delta}(\mathbf{w} \cdot \exp (\vec{z}))=\sum_{\vec{v} \in \mathbf{Z}^{g}}\left\{\prod_{i=1}^{g} P_{i i}^{\left(v_{l}^{2}-v_{i}\right) / 2} \prod_{i<j} P_{i j}^{v_{i} v_{j}} \prod_{i=1}^{g} w_{i}^{v_{l}} \sum_{n=0}^{\infty} \frac{1}{n !}\left(\sum_{i=1}^{g} v_{i} z_{i}\right)^{n}\right\}
$$

and

$$
\tau(\mathbf{t})=\exp \left(\frac{1}{2} \sum_{n, m=1}^{\infty} Q_{n m} t_{n} t_{m}\right) \cdot \Theta_{\delta}\left(\mathbf{w} \cdot \exp \left(\sum_{m=1}^{\infty} t_{m} \vec{R}_{m}\right)\right) .
$$

Then by Proposition 3.2,

$$
\begin{aligned}
\left.\tau_{\delta}(\mathbf{t})\right|_{y_{1}=\cdots=y_{g}=0}= & \sum_{\vec{v} \in\{0,1\}^{g}}\left[\prod_{i<j}\left[x_{i}, x_{-i}, x_{j}, x_{-j}\right]^{v_{i} v_{j}} \prod_{i=1}^{g} w_{i}^{v_{l}}\right. \\
& \left.\times \sum_{n=0}^{\infty} \frac{1}{n !}\left\{\sum_{i=1}^{g} v_{i} \sum_{m=1}^{\infty}\left(\frac{1}{\left(x_{-i}-p\right)^{m}}-\frac{1}{\left(x_{i}-p\right)^{m}}\right) t_{m}\right\}^{n}\right]
\end{aligned}
$$

which induces the soliton solution (cf. [Go, Mum2]).

\section{4. $P$-adic Solutions of $K P$}

4.1. Let $K$ be a complete nonarchimedean valuation field, and let $\Gamma$ be a Schottky group of rank $g$ over $K$. Then as seen in 2.1 (cf. [Ge]), there exist free generators $\gamma_{1}, \ldots, \gamma_{g}$ of $\Gamma$ whose Koebe coordinates $\left(\alpha_{ \pm k}, \beta_{k}\right)_{1 \leqq k \leqq g}$ satisfy $\alpha_{i} \neq \alpha_{j}(i \neq j)$ and

$$
\left|\beta_{k}\right|<\min \left\{\left|\left[\alpha_{k}, \alpha_{-k} ; \alpha_{i}, \alpha_{j}\right]\right| ; i, j \neq \pm k\right\} \quad(k=1, \ldots, g) .
$$

Taking the conjugation $\gamma_{i} \mapsto \rho \gamma_{i} \rho^{-1}(i=1, \ldots, g)$ by a certain $\rho \in P G L_{2}(K)$, we may assume that $\alpha_{ \pm 1}, \ldots, \alpha_{ \pm g} \neq \infty$. Then we can take $D_{ \pm 1}, \ldots, D_{ \pm g}$ as in 2.1 such

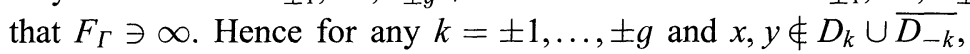

$$
\left|\frac{\alpha_{k}-x}{\alpha_{-k}-x} \beta_{k}\right|,\left|\left[\alpha_{k}, \alpha_{-k} ; x, y\right] \beta_{k}\right|<1 \text {. }
$$

Put

$$
r=\max \left\{\left|\frac{\alpha_{k}-\alpha_{i}}{\alpha_{-k}-\alpha_{i}} \beta_{k}\right|,\left|\left[\alpha_{k}, \alpha_{-k} ; \alpha_{i}, \alpha_{j}\right] \beta_{k}\right| ; i, j \neq \pm k\right\} .
$$

Then $r<1$.

4.2. Lemma. Let $k(1), \ldots, k(l) \in\{ \pm 1, \ldots, \pm g\}$ such that $k(s) \neq-k(s+1)(s=$ $1, \ldots, l-1)$, and put

$$
\tau=\frac{\prod_{s=2}^{l}\left(\alpha_{k(s)}-\alpha_{-k(s-1)}\right)}{\prod_{s=1}^{l}\left(\alpha_{k(s)}-\alpha_{-k(s)}\right)}
$$




\section{Then}

(a) For any $x, y \in F_{\Gamma}$,

$$
\left|\frac{\prod_{s=1}^{l} \beta_{k(s)}}{\tau^{2}\left(\alpha_{k(1)}-x\right)\left(\alpha_{-k(l)}-y\right)}\right|<r^{l-2} .
$$

(b) For any $j \neq \pm k(l)$ and $z \in F_{\Gamma}$,

$$
\left|\frac{\left(\alpha_{j}-\alpha_{-j}\right) \prod_{s=1}^{l} \beta_{k(s)}}{\tau^{2}\left(\alpha_{j}-\alpha_{-k(l)}\right)\left(\alpha_{-j}-\alpha_{-k(l)}\right)\left(\alpha_{k(1)}-z\right)}\right|<r^{l-1} .
$$

(c) For any $i \neq \pm k(1)$ and $j \neq \pm k(l)$,

$$
\left|\frac{\left(\alpha_{i}-\alpha_{-i}\right)\left(\alpha_{j}-\alpha_{-j}\right) \prod_{s=1}^{l} \beta_{k(s)}}{\tau^{2}\left(\alpha_{k(1)}-\alpha_{i}\right)\left(\alpha_{k(1)}-\alpha_{-i}\right)\left(\alpha_{j}-\alpha_{-k(l)}\right)\left(\alpha_{-j}-\alpha_{-k(l)}\right)}\right| \leqq r^{l} .
$$

Proof. We prove only (a) because one can show (b) and (c) in the same way. Since

$$
\begin{aligned}
\left|\frac{\alpha_{k(1)}-\alpha_{-k(1)}}{\alpha_{k(1)}-x}\right| \leqq \max \left\{1,\left|\frac{\alpha_{-k(1)}-x}{\alpha_{k(1)}-x}\right|\right\}, \\
\left|\frac{\alpha_{k(s)}-\alpha_{-k(s)}}{\alpha_{k(s+1)}-\alpha_{-k(s)}}\right| \leqq \max \left\{1,\left|\frac{\alpha_{k(s)}-\alpha_{k(s+1)}}{\alpha_{-k(s)}-\alpha_{k(s+1)}}\right|\right\} \quad(s=1, \ldots, l-1), \\
\left|\frac{\alpha_{k(s)}-\alpha_{-k(s)}}{\alpha_{k(s)}-\alpha_{-k(s-1)}}\right| \leqq \max \left\{1,\left|\frac{\alpha_{-k(s)}-\alpha_{-k(s-1)}}{\alpha_{k(s)}-\alpha_{-k(s-1)}}\right|\right\} \quad(s=2, \ldots, l), \\
\left|\frac{\alpha_{k(l)}-\alpha_{-k(l)}}{y-\alpha_{-k(l)}}\right| \leqq \max \left\{1,\left|\frac{\alpha_{k(l)}-y}{\alpha_{-k(l)}-y}\right|\right\},
\end{aligned}
$$

we have

$$
\left|\frac{\prod_{s=1}^{l} \beta_{k(s)}}{\tau^{2}\left(\alpha_{k(1)}-x\right)\left(y-\alpha_{-k(l)}\right)}\right| \leqq \prod_{s=1}^{l} v_{k(s)}
$$

where

$$
\begin{aligned}
v_{k(1)}= & \left|\beta_{k(1)}\right| \max \left\{1,\left|\frac{\alpha_{-k(1)}-x}{\alpha_{k(1)}-x}\right|,\left|\frac{\alpha_{k(1)}-\alpha_{k(2)}}{\alpha_{-k(1)}-\alpha_{k(2)}}\right|,\right. \\
& \left.\left|\left[\alpha_{k(1)}, \alpha_{-k(1)} ; \alpha_{k(2)}, x\right]\right|\right\}, \\
v_{k(s)}= & \left|\beta_{k(s)}\right| \max \left\{1,\left|\frac{\alpha_{k(s)}-\alpha_{k(s+1)}}{\alpha_{-k(s)}-\alpha_{k(s+1)}}\right|,\left|\frac{\alpha_{-k(s)}-\alpha_{-k(s-1)}}{\alpha_{k(s)}-\alpha_{-k(s-1)}}\right|,\right. \\
& \left.\left|\left[\alpha_{k(s)}, \alpha_{-k(s)} ; \alpha_{k(s+1)}, \alpha_{-k(s-1)}\right]\right|\right\} \quad(s=2, \ldots, l-1), \\
v_{k(l)}= & \left|\beta_{k(l)}\right| \max \left\{1,\left|\frac{\alpha_{-k(l)}-\alpha_{-k(l-1)}}{\alpha_{k(l)}-\alpha_{-k(l-1)}}\right|,\left|\frac{\alpha_{k(l)}-y}{\alpha_{-k(l)}-y}\right|,\right. \\
& \left.\left|\left[\alpha_{k(l)}, \alpha_{-k(l)} ; y, \alpha_{-k(l-1)}\right]\right|\right\},
\end{aligned}
$$

which implies (a). 
4.3. Theorem. Let $\left(\Gamma ; \gamma_{1}, \ldots, \gamma_{g}\right)$ and $\left(\alpha_{ \pm k}, \beta_{k}\right)_{1 \leqq k \leqq g}$ be as in 4.1 , let $p$ be a point of $F_{\Gamma}-\{\infty\}$, and put $u=z-p$. Then

(a) For any $n \geqq 0$,

$$
\omega^{(n)}=\sum_{\gamma \in \Gamma} \frac{\gamma^{\prime}(z)}{(\gamma(z)-p)^{n+1}} d u
$$

is uniformly convergent for $z \in H_{\Gamma}-\cup_{\gamma \in \Gamma} \gamma(\{p, \infty\})\left(H_{\Gamma}-\cup_{\gamma \in \Gamma} \gamma(p)\right.$ if $\left.n \geqq 1\right)$ in the wider sense, and any coefficient of $\omega^{(n)} \in K[[u]] d u$ is convergent in $K$.

(b) For any $j=1, \ldots, g$,

$$
\omega_{j}=\sum_{\gamma \in \Gamma}\left(\frac{1}{z-\gamma\left(\alpha_{j}\right)}-\frac{1}{z-\gamma\left(\alpha_{-j}\right)}\right) d u
$$

is uniformly convergent for $z \in H_{\Gamma}$ in the wider sense, and any coefficient of

$$
\omega_{j}=\sum_{m=1}^{\infty} \sum_{k=1}^{m} \sum_{\gamma \in \Gamma_{j}}\left(\frac{\gamma\left(\alpha_{j}\right)-\gamma\left(\alpha_{-j}\right)}{\left(\gamma\left(\alpha_{j}\right)-p\right)^{k}\left(\gamma\left(\alpha_{-j}\right)-p\right)^{m+1-k}}\right) u^{m-1} d u
$$

in $K[[u]] d u$ is convergent in $K$.

(c) For any $i, j=1, \ldots, g, p_{i j}=\Pi_{\gamma \in \Gamma_{i j}} \psi_{i j}(\gamma)$ is convergent.

Proof. (a) Let $f=f_{k(1)} f_{k(2)} \cdots f_{k(l)}$ such that $k(s) \neq-k(s+1)(s=1, \ldots, l-1)$, and put

$$
\left(\begin{array}{ll}
a & b \\
c & d
\end{array}\right)=\left.f\right|_{x_{ \pm k}=\alpha_{ \pm k}, y_{k}=\beta_{k}}
$$

Then in the same way as the proof of Proposition 3.2(d), one can show that

$$
\begin{aligned}
& \left.f\right|_{x_{ \pm k}=\alpha_{ \pm k}, y_{k}=\beta_{k}}=\tau\left\{\left(\begin{array}{cc}
\alpha_{k(1)} & -\alpha_{k(1)} \alpha_{-k(l)} \\
1 & -\alpha_{-k(l)}
\end{array}\right)\right. \\
& -A \frac{\alpha_{k(2)}-\alpha_{k(1)}}{\alpha_{k(2)}-\alpha_{-k(1)}} \beta_{k(1)}\left(\begin{array}{cc}
\alpha_{-k(1)} & -\alpha_{-k(1)} \alpha_{-k(l)} \\
1 & -\alpha_{-k(l)}
\end{array}\right) \\
& -B \frac{\alpha_{-k(l)}-\alpha_{-k(l-1)}}{\alpha_{k(l)}-\alpha_{-k(l-1)}} \beta_{k(l)}\left(\begin{array}{cc}
\alpha_{k(1)} & -\alpha_{k(1)} \alpha_{k(l)} \\
1 & -\alpha_{k(l)}
\end{array}\right) \\
& \left.\quad+C \frac{\alpha_{k(2)}-\alpha_{k(1)}}{\alpha_{k(2)}-\alpha_{-k(1)}} \frac{\alpha_{-k(l)}-\alpha_{-k(l-1)}}{\alpha_{k(l)}-\alpha_{-k(l-1)}} \beta_{k(1)} \beta_{k(l)}\left(\begin{array}{cc}
\alpha_{-k(1)} & -\alpha_{-k(1)} \alpha_{k(l)} \\
1 & -\alpha_{k(l)}
\end{array}\right)\right\} .
\end{aligned}
$$

Here

$$
\tau=\frac{\prod_{s=2}^{l}\left(\alpha_{k(s)}-\alpha_{-k(s-1)}\right)}{\prod_{s=1}^{l}\left(\alpha_{k(s)}-\alpha_{-k(s)}\right)}
$$

and $A, B$ and $C$ are the sums of products $\prod_{s=2}^{l-1} u_{s}$, where each $u_{s}$ is either 1 or one of the following:

$$
-\left[\alpha_{k(s)}, \alpha_{-k(s)} ; \alpha_{ \pm k(s+1)}, \alpha_{ \pm k(s-1)}\right] \beta_{k(s)}, \quad-\left[\alpha_{k(s)}, \alpha_{-k(s)} ; \alpha_{ \pm k(s+1)}, \alpha_{\mp k(s-1)}\right] \beta_{k(s)} .
$$


Hence $|A|,|B|,|C| \leqq 1$. Since

$$
\begin{aligned}
c z+d= & \tau\left(z-\alpha_{-k(l)}\right)\left\{1-A \frac{\alpha_{k(2)}-\alpha_{k(1)}}{\alpha_{k(2)}-\alpha_{-k(1)}} \beta_{k(1)}\right. \\
& -B \frac{\alpha_{-k(l)}-\alpha_{-k(l-1)}}{\alpha_{k(l)}-\alpha_{-k(l-1)}} \frac{z-\alpha_{k(l)}}{z-\alpha_{-k(l)}} \beta_{k(l)} \\
& \left.+C \frac{\alpha_{k(2)}-\alpha_{k(1)}}{\alpha_{k(2)}-\alpha_{-k(1)}} \frac{\alpha_{-k(l)}-\alpha_{-k(l-1)}}{\alpha_{k(l)}-\alpha_{-k(l-1)}} \frac{z-\alpha_{k(l)}}{z-\alpha_{-k(l)}} \beta_{k(1)} \beta_{k(l)}\right\}
\end{aligned}
$$

and

$$
\begin{aligned}
& a z+b-c p z-d p \\
& =\tau\left(\alpha_{k(1)}-p\right)\left(z-\alpha_{-k(l)}\right)\left\{1-A\left[\alpha_{k(1)}, \alpha_{-k(1)} ; \alpha_{k(2)}, p\right] \beta_{k(1)}\right. \\
& -B\left[\alpha_{k(l)}, \alpha_{-k(l)} ; z, \alpha_{-k(l-1)}\right] \beta_{k(l)}
\end{aligned}
$$

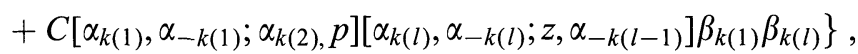

for any $z \in F_{\Gamma},|c z+d|=\left|\tau\left(z-\alpha_{-k(l)}\right)\right|$ and $|a z+b-c p z-d p|=\mid \tau\left(\alpha_{k(1)}-p\right)$ $\left(z-\alpha_{-k(l)}\right) \mid$. Therefore, by Lemma 4.2 (a),

$$
\begin{aligned}
\left|\left(\frac{f^{\prime}(z)}{(f(z)-p)^{n+1}}\right)\right|_{x_{ \pm k}=\alpha_{ \pm k}, y_{k}=\beta_{k}} \mid & =\left|\frac{(a d-b c)(c z+d)^{n-1}}{(a z+b-c p z-d p)^{n+1}}\right| \\
& =\left|\frac{\prod_{s=1}^{l} \beta_{k(s)}}{\tau^{2}\left(z-\alpha_{-k(l)}\right)^{2}\left(\alpha_{k(1)}-p\right)^{n+1}}\right| \\
& \leqq \frac{r^{l-2}}{\left|\left(z-\alpha_{-k(l)}\right)\left(\alpha_{k(1)}-p\right)^{n}\right|} \rightarrow 0 \text { if } l \rightarrow \infty .
\end{aligned}
$$

Since $\omega^{(n)}$ is $\Gamma$-invariant, it is uniformly convergent on $H_{\Gamma}-\cup_{\gamma \in \Gamma} \gamma(\{p, \infty\})$ in the wider sense. Put

$$
\begin{aligned}
& \sum_{i=0}^{\infty} s_{i} u^{i}=\left.\frac{f^{\prime}(z)}{(f(z)-p)^{n+1}}\right|_{x_{ \pm k}=\alpha_{ \pm k}, y_{k}=\beta_{k}} \\
& \quad=\frac{(a d-b c)(c p+d)^{n-1}}{\left(a p+b-c p^{2}-d p\right)^{n+1}}\left(1+\frac{c u}{c p+d}\right)^{n-1}\left(1+\frac{(a-c p) u}{a p+b-c p^{2}-d p}\right)^{-n-1} .
\end{aligned}
$$

Since $|c|=|\tau|,|c p+d|=\left|\tau\left(p-\alpha_{-k(l)}\right)\right|,|a-c p|=\left|\tau\left(\alpha_{k(1)}-p\right)\right|$ and $\mid a p+d-$ $c p^{2}-d p|=| \tau\left(\alpha_{k(1)}-p\right)\left(p-\alpha_{-k(l)}\right) \mid$,

$$
\begin{aligned}
\left|s_{i}\right| & \leqq\left|\frac{(a d-b c)(c p+d)^{n-1}}{\left(a p+b-c p^{2}-d p\right)^{n+1}}\right| \max \left\{\left|\frac{c}{c p+d}\right|^{i},\left|\frac{a-c p}{a p+b-c p^{2}-d p}\right|^{i}\right\} \\
& =\left|\frac{\prod_{s=1}^{l} \beta_{k(s)}}{\tau^{2}\left(p-\alpha_{-k(l)}\right)^{i+2}\left(\alpha_{k(1)}-p\right)^{n+1}}\right|,
\end{aligned}
$$


and hence by Lemma 4.2 (a),

$$
\left|s_{i}\right| \leqq \frac{r^{l-2}}{\left|\left(p-\alpha_{-k(l)}\right)^{i+1}\left(\alpha_{k(1)}-p\right)^{n}\right|} \rightarrow 0 \quad \text { if } l \rightarrow \infty .
$$

Therefore, any coefficient of $\omega^{(n)} \in K[[u]] d u$ is convergent.

(b) Let $f$ be as in (a), and put $\gamma=\left.f\right|_{x_{ \pm k}=\alpha_{ \pm k}, y_{k}=\beta_{k}} \bmod \left(K^{\times}\right)$. Then for $j \neq \pm k(l)$ and $i \neq-k(1)$,

$$
\begin{aligned}
\left|\gamma\left(\alpha_{ \pm j}\right)-\alpha_{-i}\right| & =\frac{\left|a \alpha_{ \pm j}+b-c \alpha_{-i} \alpha_{ \pm j}-d \alpha_{-i}\right|}{\left|c \alpha_{ \pm j}+d\right|} \\
& =\frac{\left|\tau\left(\alpha_{k(1)}-\alpha_{-i}\right)\left(\alpha_{ \pm j}-\alpha_{-k(l)}\right)\right|}{\left|\tau\left(\alpha_{ \pm j}-\alpha_{-k(l)}\right)\right|}=\left|\alpha_{k(1)}-\alpha_{-i}\right|
\end{aligned}
$$

and

$$
\left|\frac{\gamma\left(\alpha_{ \pm j}\right)-\alpha_{i}}{\gamma\left(\alpha_{ \pm j}\right)-\alpha_{-i}} \beta_{i}\right|<1
$$

because $\gamma\left(\alpha_{ \pm j}\right) \notin D_{-i}$. Therefore,

$$
\begin{aligned}
& \left|\left(\gamma_{i} \gamma\right)\left(\alpha_{j}\right)-\left(\gamma_{i} \gamma\right)\left(\alpha_{-j}\right)\right| \\
& \quad=\left|\frac{\left(\gamma\left(\alpha_{j}\right)-\gamma\left(\alpha_{-j}\right)\right)\left(\alpha_{i}-\alpha_{-i}\right)^{2} \beta_{i}}{\left(\gamma\left(\alpha_{j}\right)-\alpha_{-i}\right)\left(\gamma\left(\alpha_{-j}\right)-\alpha_{-i}\right)\left(1-\frac{\gamma\left(\alpha_{j}\right)-\alpha_{i}}{\gamma\left(\alpha_{j}\right)-\alpha_{-i}} \beta_{i}\right)\left(1-\frac{\gamma\left(\alpha_{-j}\right)-\alpha_{i}}{\gamma\left(\alpha_{-j}\right)-\alpha_{-i}} \beta_{i}\right)}\right| \\
& \quad=\left|\frac{\left(\gamma\left(\alpha_{j}\right)-\gamma\left(\alpha_{-j}\right)\right)\left(\alpha_{i}-\alpha_{-i}\right)^{2} \beta_{i}}{\left(\alpha_{k(1)}-\alpha_{-i}\right)^{2}}\right|
\end{aligned}
$$

and hence by induction on $l$, we have

$$
\left|\gamma\left(\alpha_{j}\right)-\gamma\left(\alpha_{-j}\right)\right|=\left|\frac{\left(\alpha_{j}-\alpha_{-j}\right) \prod_{s=1}^{l} \beta_{k(s)}}{\tau^{2}\left(\alpha_{j}-\alpha_{-k(l)}\right)\left(\alpha_{-j}-\alpha_{-k(l)}\right)}\right| .
$$

Since

$$
\begin{aligned}
\left|\gamma\left(\alpha_{ \pm j}\right)-z\right| & =\frac{\left|a \alpha_{ \pm j}+b-c z \alpha_{ \pm j}-d z\right|}{\left|c \alpha_{ \pm j}+d\right|} \\
& =\frac{\left|\tau\left(\alpha_{k(1)}-z\right)\left(\alpha_{ \pm j}-\alpha_{-k(l)}\right)\right|}{\left|\tau\left(\alpha_{ \pm j}-\alpha_{-k(l)}\right)\right|} \\
& =\left|\alpha_{k(1)}-z\right|
\end{aligned}
$$

for any $z \in F_{\Gamma}$, by Lemma 4.2 (b),

$$
\begin{aligned}
\left|\frac{1}{z-\gamma\left(\alpha_{j}\right)}-\frac{1}{z-\gamma\left(\alpha_{-j}\right)}\right| & =\left|\frac{\gamma\left(\alpha_{j}\right)-\gamma\left(\alpha_{-j}\right)}{\left(z-\gamma\left(\alpha_{j}\right)\right)\left(z-\gamma\left(\alpha_{-j}\right)\right)}\right| \\
& =\left|\frac{\left(\alpha_{j}-\alpha_{-j}\right) \prod_{s=1}^{l} \beta_{k(s)}}{\tau^{2}\left(\alpha_{j}-\alpha_{-k(l)}\right)\left(\alpha_{-j}-\alpha_{-k(l)}\right)\left(\alpha_{k(1)}-z\right)^{2}}\right| \\
& \leqq \frac{r^{l-1}}{\left|\alpha_{k(1)}-z\right|} \longrightarrow 0 \quad \text { if } l \rightarrow \infty
\end{aligned}
$$


Since $\omega_{j}$ is $\Gamma$-invariant, it is uniformly convergent on $H_{\Gamma}$. Similarly, one can show that

$$
\sum_{k=1}^{m} \sum_{\gamma \in \Gamma_{j}}\left(\frac{\gamma\left(\alpha_{j}\right)-\gamma\left(\alpha_{-j}\right)}{\left(\gamma\left(\alpha_{j}\right)-p\right)^{k}\left(\gamma\left(\alpha_{-j}\right)-p\right)^{m+1-k}}\right)
$$

is convergent for any $m$.

(c) Let $\gamma$ be as in (b), and assume that $j \neq \pm k(l)$ and $i \neq \pm k(1)$. Then by Lemma 4.2(c),

$$
\begin{aligned}
& \left|\left[\alpha_{i}, \alpha_{-i} ; \gamma\left(\alpha_{j}\right), \gamma\left(\alpha_{-j}\right)\right]-1\right|=\left|\frac{\left(\alpha_{i}-\alpha_{-i}\right)\left(\gamma\left(\alpha_{j}\right)-\gamma\left(\alpha_{-j}\right)\right)}{\left(\gamma\left(\alpha_{j}\right)-\alpha_{-i}\right)\left(\gamma\left(\alpha_{-j}\right)-\alpha_{i}\right)}\right| \\
& \quad=\left|\frac{\left(\alpha_{i}-\alpha_{-i}\right)\left(\alpha_{j}-\alpha_{-j}\right) \prod_{s=1}^{l} \beta_{k(s)}}{\tau^{2}\left(\alpha_{k(1)}-\alpha_{i}\right)\left(\alpha_{k(1)}-\alpha_{-i}\right)\left(\alpha_{j}-\alpha_{-k(l)}\right)\left(\alpha_{-j}-\alpha_{-k(l)}\right)}\right| \\
& \quad \leqq r^{l} \longrightarrow 0 \quad \text { if } l \rightarrow \infty,
\end{aligned}
$$

and hence $p_{i j}$ is convergent.

4.4. Corollary. $\omega_{j}(j=1, \ldots, g)$ (resp. $\left.\omega^{(n)}(n \geqq 1), \omega^{(0)}\right)$ are differential forms on $C_{\Gamma}$ of the first (resp. second, third) kind.

Proof. This follows from Theorem 4.3 and that $\omega_{j}, \omega^{(n)}$ are all $\Gamma$-invariant.

4.5. We assume that $K$ is of characteristic 0 . Let $\left(\alpha_{ \pm k}, \beta_{k}\right)_{1 \leqq k \leqq g}$ be as in 4.1 , and assume that

$$
\left|\beta_{k}\right|<\min \left\{\left|4\left[\alpha_{k}, \alpha_{-k} ; \alpha_{i}, \alpha_{j}\right]\right| ; i, j \neq \pm k\right\} \quad(k=1, \ldots, g)
$$

which is automatically satisfied if the residual characteristic of $K$ is not 2. Then by the proof of Theorem 4.3 (c),

$$
\left|\frac{1}{4}\left(\prod_{\gamma \in \Gamma_{i i}-\{1\}} \psi_{i i}(\gamma)-1\right)\right|<1 \quad(i=1, \ldots, g) .
$$

For each $i=1, \ldots, g$, fix square roots $\beta_{i}^{1 / 2}$ of $\beta_{i}$, and assume that $\beta_{i}^{1 / 2} \in K^{\times}$. Then

$$
\begin{aligned}
& \beta_{i}^{1 / 2}\left\{\sum_{n=0}^{\infty}\left(\begin{array}{c}
-1 / 2 \\
n
\end{array}\right)\left(\prod_{\gamma \in \Gamma_{i i}-\{1\}} \psi_{i i}(\gamma)-1\right)^{n}\right\}^{-1} \\
& =\beta_{i}^{1 / 2}\left\{\sum_{n=0}^{\infty}\left(\begin{array}{c}
2 n \\
n
\end{array}\right)\left(-\frac{1}{4}\right)^{n}\left(\prod_{\gamma \in \Gamma_{i i}-\{1\}} \psi_{i i}(\gamma)-1\right)^{n}\right\}^{-1}
\end{aligned}
$$

is convergent and a square root of $p_{i i}$ which we denote by $p_{i i}^{1 / 2}$. Let $\left(\Gamma ; \gamma_{1}, \ldots, \gamma_{g}\right)$ be the marked Schottky group over $K$ with Koebe coordinates $\left(\alpha_{ \pm k}, \beta_{k}\right)_{1 \leqq k \leqq g}$, let $P$ be the point of $C_{\Gamma}$ corresponding to a point $p$ of $F_{\Gamma}-\{\infty\}$, and put $u=z-p$. Then from $X=\left(C_{\Gamma}, P, u\right)$, we construct a micro-differential operator as follows. By 
Theorem 4.3 (b), there exist uniquely $q_{n m}, r_{j m} \in K$ such that

$$
\begin{aligned}
\omega^{(n)} & =\left(\frac{1}{u^{n+1}}+\sum_{m=1}^{\infty} \frac{q_{n m}}{n} u^{m-1}\right) d u \quad(n \geqq 0), \\
\omega_{j} & =\sum_{m=1}^{\infty} r_{j m} u^{m-1} d u \quad(j=1, \ldots, g) .
\end{aligned}
$$

Since $\log \left|\Pi_{i=1}^{g} p_{i i}^{v_{i}^{2} / 2} \Pi_{i<j} p_{i j}^{v_{i} v_{j}}\right|$ is a negative definite form for $\vec{v}=\left(v_{i}\right)_{1 \leqq i \leqq g} \in \mathbf{Z}^{g}$, (cf. [M-D], Sect. 4), for $\mathbf{c}=\left(c_{i}\right)_{1 \leqq i \leqq g} \in\left(K^{\times}\right)^{g}$ and a vector $\vec{z}=\left(z_{i}\right)_{1 \leqq i \leqq g}$ of $g$ indeterminates,

$$
\Theta(\mathbf{c} \cdot \exp (\vec{z}))=\sum_{\vec{v} \in \mathbf{Z}^{g}}\left\{\prod_{i=1}^{g} p_{i i}^{v_{i}^{2} / 2} \prod_{i<j} p_{i j}^{v_{l} v_{j}} \prod_{i=1}^{g} c_{i}^{v_{i}} \sum_{n=0}^{\infty} \frac{1}{n !}\left(\sum_{i=1}^{g} v_{i} z_{i}\right)^{n}\right\}
$$

belongs to $K\left[\left[z_{1}, \ldots, z_{g}\right]\right]$. We define the $\tau$-function for $(X, \mathbf{c})$ by

$$
\tau\left(\mathbf{t}, X_{\mathbf{c}}\right)=\exp \left(\frac{1}{2} \sum_{n, m=1}^{\infty} q_{n m} t_{n} t_{m}\right) \Theta\left(\mathbf{c} \cdot \exp \left(\sum_{m=1}^{\infty} t_{m} \vec{r}_{m}\right)\right)
$$

where $\vec{r}_{m}=\left(r_{j m}\right)_{1 \leqq j \leqq g}$. Then as in (1.4)-(1.6), for any $\mathbf{c} \in\left(K^{\times}\right)^{g}$ with $\Theta(\mathbf{c}) \neq 0, \tau\left(\mathbf{t}, X_{\mathbf{c}}\right)$ defines a micro-differential operator with coefficients in $K[[x, \mathbf{t}]]$ which we denote by $L\left(\mathbf{t}, X_{\mathbf{c}}\right)$.

\subsection{Theorem.}

(a) $L\left(\mathbf{t}, X_{\mathbf{c}}\right)=\left.L(\mathbf{t})\right|_{x_{ \pm k}=\alpha_{ \pm k}, y_{k}^{1 / 2}=\beta_{k}^{1 / 2}, w_{k}=c_{k}}$.

(b) $L\left(\mathbf{t}, X_{\mathbf{c}}\right)$ satisfies the KP hierarchy (1.7). In particular,

$$
u_{1}\left(x, t_{2}, t_{3}\right)=\frac{\partial^{2}}{\partial x^{2}} \log \Theta\left(\mathbf{c} \cdot \exp \left(x \vec{r}_{1}+t_{2} \vec{r}_{2}+t_{3} \vec{r}_{3}\right)\right)+q_{11}
$$

satisfies the KP equation (1.8).

Proof. This follows from the definition of $L\left(\mathbf{t}, X_{\mathbf{c}}\right)$ and Theorem 3.4 (b).

\subsection{Remark. Since}

$$
\tau\left(\mathbf{t}, X_{\mathbf{c}}\right)=\left.\tau(\mathbf{t})\right|_{x_{ \pm k}=\alpha_{ \pm k}, y_{k}^{1 / 2}=\beta_{k}^{1 / 2}, w_{k}=c_{k}},
$$

for any $\mathbf{c} \in\left(K^{\times}\right)^{g}$, it is easy to see that $\tau\left(\mathbf{t}, X_{\mathbf{c}}\right)$ and

$$
L\left(\mathbf{t}, X_{\mathbf{c}}\right)=\left.L(\mathbf{t})\right|_{x_{ \pm k}=\alpha_{ \pm k}, y_{k}^{1 / 2}=\beta_{k}^{1 / 2}, w_{k}=c_{k}}
$$

satisfy the same relation as in (1.4)-(1.6) and that $L\left(\mathbf{t}, X_{\mathbf{c}}\right)$ satisfies (1.7).

4.8. Remark. One can extend Theorem 4.6 for general local coordinates $u$ without difficulty. 


\section{References}

[A] Akaza, T.: Poincare theta series and singular sets of Schottky groups. Nagoya Math. J. 24, 43-65 (1964)

[A-D] Arbarello, E., De Concini, C.: On a set of equations characterizing Riemann matrices. Ann. Math. 120, 119-140 (1984)

[Ge] Gerritzen, L.: Zur analytischen Beschreibung des Raumes der Schottky-MumfordKurven. Math. Ann. 255, 259-271 (1981)

[Go] González-diéz, G.: Theta functions on the boundary of moduli space. In: Donagi, R. (ed.) Curves, Jacobians, and Abelian Varieties, Contemp. Math. 136, Providence RI: Amer. Math. Soc., 1992, pp. 185-208

[G-v.d.P] Gerritzen, L., van der Put, M.: Schottky groups and Mumford curves, Lect. Notes Math. 817, Berlin, Heidelberg, New York: Springer, 1980

[H] Hejhal, D.A.: On Schottky and Teichmüller spaces. Adv. Math. 15, 133-156 (1975)

[I] Ichikawa, T.: The universal periods of curves and the Schottky problem. Compos. Math. 85, 1-8 (1993)

[Ko] Koebe, P.: Über die Uniformisierung der algebraischen Kurven IV. Math. Ann. 75, 42-129 (1914)

[Kr] Krichever, I.M.: Methods of algebraic geometry in the theory of nonlinear equations. Russ. Math. Surv. 32, 185-214 (1977)

[M-D] Manin, Y., Drinfeld, V.: Periods of $p$-adic Schottky groups. J. Reine Angew. Math. 262/263, 239-247 (1973)

[Mul] Mulase, M.: Cohomological structure in soliton equations and jacobian varieties. J. Differential Geom. 19, 403-430 (1984)

[Mum1] Mumford, D.: An analytic construction of degenerating curves over complete local fields. Compos. Math. 24, 129-174 (1972)

[Mum2] Mumford, D.: Tata lectures on theta II, Progress in Math. 43, Basel: Birkhäuser, 1983

[S-S] Sato, M., Sato, Y.: Soliton equations as dynamical systems on infinite dimensional Grassmann manifold. Lax, P.D. (ed.) Nonlinear Partial Differential Equations in Applied Sciences, Lect. Notes Num. Appl Anal. 5, Amsterdam: North-Holland and Tokyo: Kinokuniya, 1982, pp. 259-271

[Sc] Schottky, F.: Über eine spezielle Funktion, welche bei einer bestimmten linearen Transformation ihres Arguments unverändert bleibt. J. Reine Angew. Math. 101, 227-272 (1887)

[Sh] Shiota, T.: Characterization of jacobian varieties in terms of soliton equations. Invent. Math. 83, 333-382 (1986)

Communicated by M. Jimbo 
